\title{
THE CLASSIFICATION OF QUADRICS*
}

\author{
$\mathrm{BI}$
}

\section{T. J. I'A. BRONWICH}

In a former number of the Transactions $\nmid$ Mr. J. L. Coolidge has given a classified list of the possible species of quadrics in hyperbolic space; his method is based on that used by CLEBsCr for discussing the mutual relations of two quadrics. It seems desirable, however, that the classification should be associated with the algebraic problem of reducing two quaternary quadratic forms to canonical types; the solution of this problem is, of course, based on WerersTrass's theory of increriant fiectors. $\neq$ One obvious advantage of using invariant factors as a basis of classification is that they can be determined without any preliminary recluction of the equation to the quadric; indeed, it is generally necessary to find the factor's before we can begin the reduction. Another advantage may be found in the fact that the canonical types given by $W_{\text {EIER- }}$ srrass are the most compact possible.\$

The first classification of two quadries by means of invariant factors is due to Sylvester. || From the geometrical point of view, an elaborate discussion was carried ont, independently of Weiensriass's methods, by Painvin; $\uparrow$ Painvin touches on the question of reality, but only in the simplest case (when the invariant factors are linerer). According to Muti, the application to quadrics

* Presented to the Society at the St. Louis meeting, September 17, 1904. Receired for publication March 4, 1904.

†Transactions of the American Mathematical Society, vol. 4 (1903), p. 161.

$\ddagger$ Elementartheiler; see his paper on bilinear and quadratic forms, Ferliner ll onatsberich te, 1868 , p. $310=$ Cestmmelte Werle', vol. 2, p. 19. For a bibliography, consult a review of IIUTH's Flementurtheiler (Leipzig, 1899), in the Bulletin of the American Mathematical Society, vol. $\tau$ (1901), p. 308. An elementary account of the application of this theory to the reduction of two quadratic forms will be found in a paper by the writer in the Quarterly Journal of Mathematics, vol. $33(1901)$, p. 85.

?. As we shall see later, some of Confubik's types contain superfluous coefficients, which uay be removed by suitable linear substitutions.

IPhilosophical Iragazine, ser. 4, vol. 1 (1851), pp. 119, 295; this paper is, of course, earlier than WEIERSTR LSs's. But it is not complete, as no method for determining the reducing substitutions is given, and thus it is not certain that the classification is exhaustive. In fact the two "singular cases" are omitted; i. e., when the quadrics are cones having (i) a common tangent-plane, which they touch along a common generator (ii) a common vertex.

Touvelles Annales de Mathémati i ues, ser. 2, vol. 7 (1868), pp. 481, 529); vol. $8(1869)$, pp. 49, 145, 193. The last section of his paper contains a list of the chief results obtained. 
of Weierstrass's results (and of Kronecker's," on the "singular case") has been fully carried out by Kinling. $\dagger$

Weinerstrass's method of determining the reducing substitutions does not show how to modify them in case we restrict ourselves to real quadratic forms and real linear substitutions. This difficulty was first surmounted by KLEIN in his celebrated memoir on the classification of line-complexes of the second degree $\ddagger$; KLEIN shows there that if one of the quadratic forms has a known type, and if we are restricted to real forms (and real substitutions), then the possible invariant-factors are restricted by a certain relation. $\|$ KL.FIN gives full details of all possible cases, if the number of variables is six, and the signature of the one known quadratic form is zero (see $\S 1$ below, for definition of signature).

BôcuER $§$ has carried out a similar classification for the case of five variables, with a quadratic form of signature three.

In what follows we shall consider the case of four variables, the given signature being two (\$2), four $(\$ 3)$, or three $(\S 4)$.

\section{§1. Preliminary results on Beal transformations of quadrutic forms.}

Any real quadratic form can be expressed in the shape

$$
a_{1} x_{1}^{2}+a_{2} x_{2}^{2}+\cdots+a_{n} x_{n}^{2}
$$

(where $x_{1}, x_{2}, \cdots, x_{n}$ are real and linearly independent) in in infinity of ways; but, however the reduction is effected, the number of positive coefficients and the number of negative coefficients (in $a_{1}, a_{2}, \ldots, a_{11}$ ) are both fixed. This is Sylvester's lav of inertia (Trügheitsgesetz) of quadratic forms. 9

* Kronecker published a number of papers on this case; a list would be too long for enumeration here. The earlier papers will be found in the second half of rolume 1 of his works; the last paper (in which the question was finally settled) appeared in the Berliner Sitzungsberichte of 1890,1891 . Alternative methods have been given ly the present writer, Proceedings of the London Mathematical Society, vol. 32 (1900), pp. 87, 117, 326.

$\dagger$ Der Flächenbüschel zueiter Ordnung, Incuguraldissertution, Berlin, 187:2; unfortunately this memoir has not been accessible. It does not appear from IIUTH's references, whether or no KILLING concerns himself with the question of reality.

† Inauguraldissertution, Bonn, 1868; reprinted in vol. 23 of the $M$ athematische Annalen, 1. 539.

UI Mthematische Annalen, vol. 23, pp. 561, 562 ; the theorem has been extenden to "Heknite's forms" by A. Loewy, Crelle's Journal für die Matbematik, vol. 122 (1900), p 69; see also a paper by the writer, Proceedings of the London Mathematical Society. vol. 32 (1901), p. 349.

\& Reihenentuickelungen der Potentialtheorie, Leipzig, 1894 ; the classification (of cyclides) will be found in Kapitel 3. It is perhaps of interest to note that the results obtained in $\$ 2$ below can also be used to classify the cyclic curves (i. e. bicircular quartics), mentioned by BûcHER.

I Philosophical Magazine, ser. 4, vol. 2(1852), p. 138; Philosophical Transactions of London, 1853, p. 407. Borchari) (Crelle's Jourual fürdie Mathematik, 
For our present purpose, the difference between these two numbers i.; the most useful constant; this difference we call the signature of the quadratic form, after Froberius.*

We are now in a position to state KLeIs's theorem (given in the dissertation alreatly cited) :-

If $A$ is a real quadratic form, whose signature is known to be m, then, whatecer quadratic form $B$ may be (prorided that it is real), the characteristic aterminut $|\lambda A-B|$ has at lecust m REAL invariant factors of ODD elegree. $\dagger$

In the present application, $A$ is the absolute quadric (of hyperbolic space) and takes the form

$$
x_{1}^{2}+x_{2}^{2}+x_{3}^{2}-x_{1}^{2}
$$

so that its signature is 2(i. e. $3-1$ ). Accordingly, whatever quadric $B$ may be, the determinant $\left|\lambda_{A} 1-B\right|$ has at least two real invariant-factors of odd degree, Now the degree of this determinant is $\mathbf{4}$, so that the two real invariant-factors must have degrees 1,1 or 3,1 . Hence the only possible combinations of indices for the invariant-factors are given by the sets

$$
1111 ; 211 ; 31 \text {. }
$$

It is now clear that complex roots can occur only in the first set ; further, in this set there may be one pair of such roots (not more). Thus the four fundamental types are

$\begin{array}{cccc}\text { I } & \text { II } & \text { III } & \text { IV } \\ 1111 & 1111 & 211 & 31\end{array}$

vol. 53 (1857), p. 275 ) points ont that $\mathrm{JACOBI}$ was acquainted with the result as early as 1847 ; buc apparently $J_{A C O B I}$ had no occasion to publish it.

The restrictions that $x_{1}, x_{2}, \cdots, x_{n}$ must be real and linearly independent are essential ; for example, we have

$$
(x+i y)^{2}+(x-i y)^{2}=2 x^{2}-2 y^{2} \text {, and } x^{2}+y^{2}+(x+y)^{2}+(x-y)^{2}=3 x^{2}+3 y^{2} .
$$

* Crelle's Journal für die Mathematik, vol. 114 (1894), p. 187 : the paper was also published in the Berliner Sit zungsberichte of that year.

† This theorem requires modification in case $|A|=0$. For instance, if there are three variables $x, y, z$ and if $A=x^{2}+y^{2}, B=2 y z$, the signature of $A$ is 2 , but there is only one invariantfactor of $|\lambda A+\mu B|$ which has an odd degree ; the invariant-factors are actually $i, \mu^{2}$. Of course in KLEIN's work the case $|A|=0$ is excluded owing to the nature of the problem.

The modified theorem may be stated as follows: Let $|i A+\mu B|$ hare $n_{1}$ inrariant-factors of the type $(\lambda+\mu c) \gamma, c$ being real (including zero) and $;$ being odd ; and let $n_{2}$ be the number of incariantfactors of the type $\mu \beta$ where $;$ is ecen; then

$$
n_{1}+n_{2} \geqq m \text {. }
$$

In the illustration above we have $n_{1}=1, n_{2}=1$, and the theorem is verified. For a discussion of the last theorem, see A. Loeivy, Crelle's Journal für die Mathematik, vol. 122 (1900), p. 61 ; Bronwref, Proceedings of the London Mathematical Society, vol. $32(1900)$, p. 162. 
where, in case II, we have one pair of conjugate complex roots, indicated by the bar over the third and fourth indices.

We can now write down the standard types of terms which occur in the reduced* equations to the quadrics $A, B$; lists of these types will be found in the papers already cited and in Mutr's Elementurtheiler.

Let $c$ be the typical real root of the determinant $|\lambda A-B|$, then the standard types are given by the following table :-

\begin{tabular}{c|c|c|c} 
Invariant-factor & $\lambda-c$ & $(\lambda-c)^{2}$ & $(\lambda-c)^{3}$ \\
\hdashline Terms in $A$ & $\pm x^{2}$ & $2 x y$ & $2 x y+z^{2}$ \\
\hdashline Terms in $B$ & $\pm c x^{2}$ & $2 c x y \pm x^{2}$ & $c\left(2 x y+z^{2}\right)+2 z x$
\end{tabular}

where $x, y, z$ are all real and do not appear in any other terms.

Some modifications must, however, be introduced, in order to retain the regular type for the absolute $A$ : and so we put (in the second and third cases) $x=\xi-\eta, 2 y=\xi+\eta$, obtaining the table :-

\begin{tabular}{l:cc} 
Invariant-factor & $(\lambda-c)^{2}$ & $(\lambda-c)^{3}$ \\
\hdashline Terms in $A$ & $\xi^{2}-\eta^{2}$ & $\xi^{2}-\eta^{2}+z^{2}$ \\
\hline Terms in $B$ & $c\left(\xi^{2}-\eta^{2}\right) \pm(\xi-\eta)^{2}$ & $c\left(\xi^{2}-\eta^{2}+z^{2}\right)+2 z(\xi-\eta)$
\end{tabular}

Another change is necessary, when we have a pair of complex roots; if these are $\alpha+i \beta, \alpha-i \beta$, the corresponding reduced terms will be given by:-

\begin{tabular}{l|c} 
Invariant-factor & $(\lambda-\alpha)^{2}+\beta^{2}$ \\
\hline Terms in $A$ & $\frac{1}{2}(x+i y)^{2}+\frac{1}{2}(x-i y)^{2}=x^{2}-y^{2}$ \\
\hline Terms in $B$ & $\frac{1}{2}\left[(\alpha-i \beta)(x+i y)^{2}+(\alpha+i \beta)(x-i y)^{2}\right]=\alpha\left(x^{2}-y^{2}\right)+2 \beta x y$
\end{tabular}

where $x$ and $y$ are real.

We have now sufficient material to write down all the types of quadrics by inspection. $\dagger$

§2. Canonical forms for the possible quadrics in hyperbolic space. Type I. Indices 1111.

$$
\begin{aligned}
& A=y_{1}^{2}+y_{2}^{2}+y_{3}^{2}-y_{4}^{2}, \\
& B=c_{1} y_{1}^{2}+c_{2} y_{2}^{2}+c_{3} y_{3}^{2}-c_{4} y_{4}^{2},
\end{aligned}
$$$$
|B|=-c_{1} r_{2} c_{3} c_{4}
$$

where $c_{1}, c_{2}, c_{3}, c_{4}$ are roots of the determinantal equation in $\lambda,|\lambda A-B|=0$.

* It is to be borne in mind that the invariant-factors can be calculated from the equation to $B$, given in any form; indeed they must, in general, be found before the reduction can be carried out.

+ Since $|A|=-1$, there is here no reason to put down the types corresponding to the identical vanishing of $|\% A-B|$; nor to an infinite root of $|\lambda A-B|=0$. 
$\mathrm{I}(a) . c_{1}, c_{2}, c_{3}, c_{1}$ are all different, represented by the symbol [1111]. This case gives the ellipsoid and four hyperboloids found by Coolidge* (pp. 162, 163, l. c.).

$\mathrm{I}(b) \cdot c_{1}=c_{2}$ or $c_{3}=c_{4}$, represented by $[(11) 11]$. The case $c_{1}=c_{2}$ gives the spheroids and hyperboloids of revolution (C., p. 168); $c_{3}=c_{4}$ gives the surfaces of translation (C., § 4, p. 169).

$\mathrm{I}(c) . c_{1}=c_{2}=c_{3}$ or $c_{1}=c_{2}=c_{4}$; represented by $[(111) 1]$. These cases appear as a sphere and an equidistcent surface, respectively (C., p. 170).

$\mathrm{I}(d) \cdot c_{1}=c_{2}$ and $c_{3}=c_{4}$; represented by $[(11)(11)]$. This gives the anclogue of Clifford's surface in elliptic space.(C., end of $\S 3$, p. 169).

$\mathrm{I}(e) . \quad c_{1}=c_{2}=c_{3}=c_{4}$; represented by $[(1111)]$. This case is trivial, geometrically, as the quadric coincides with the absolute; it is, of course, algebraically distinct.

Type II. Indices 1111.

$$
\begin{aligned}
& A=y_{1}^{2}+y_{2}^{2}+y_{3}^{2}-y_{4}^{2}, \\
& B=c_{1} y_{1}^{2}+c_{2} y_{2}^{2}+\alpha\left(y_{3}^{2}-y_{4}^{2}\right)+2 \beta y_{3} y_{4}, \quad|B|=-c_{1} c_{2}\left(a^{2}+\beta^{2}\right),
\end{aligned}
$$

where the roots of $|\lambda A-B|=0$ are $c_{1}, c_{2}, \alpha+i \beta, \alpha-i \beta$.

$\mathrm{II}(a) . c_{1}, c_{2}$ are unequal; represented by $\left[\begin{array}{lll}11 & 11\end{array}\right]$. This is the surface with imaginary centers given by CoolidGE (C., p. 165). But it may be well to point out that the apparently more general expression given by him is not actually more general than this (it contains five coefficients, one of which can be removed by linear transformations) $\dagger$; and further, his equation has the disadvantage that its coefficients cannot be put down directly from the determinant $|\lambda A-B|$, before reduction.

II $(b) . c_{1}=c_{2}$; represented by $[(11) 11]$. This is a corresponding surface of revolution (C. $\S 3$, sub-case 4 ); here Coolidge's equation has the right number of arbitrary coefficients, but again they are not those naturally suggested by the determinant $|\lambda A-B|$; namely, $x=\frac{1}{2}\left(a_{3}-1\right), \beta=\frac{1}{2}\left[b^{2}-\left(a_{3}-1\right)^{2}\right]^{2}$.

* I do not stay to repeat CoolidiE's sub-classitication, which depends on the signs and relative magnitudes of the c's; and, in future, I quote his paper by the letter $C$.

† More exactly, two of them can be made equal, thus : Let $p$ be that root of the quadratio $p+p^{-1}=4 b\left(a_{3}+a_{1}\right)^{-1}$, which is numerically less than 1 . Write

then we find

$$
\begin{gathered}
x=! 2\left(a_{3}-a_{4}\right), \quad ;=1\left(a_{3}+a_{4}\right)\left(1-p^{2}\right) p^{-1}, \\
y_{3}=\left(x_{3}-p x_{1}\right)\left(1-p^{2}\right)^{-\frac{1}{2}}, \quad y_{4}=\left(p x_{3}-x_{4}\right)\left(1-p^{2}\right)^{-1} ;
\end{gathered}
$$

where

$$
i_{3}^{2}-r_{1}^{2}=y_{3}^{2}-y_{1}^{2}, \quad u_{3} x_{3}^{2}-2\left(x_{3} x_{4}+u_{4} x_{4}^{2}=\alpha\left(y_{3}^{2}-y_{1}^{2}\right)+23 y_{3} y_{2,} ;\right.
$$

$$
x \cdots \frac{1}{2}\left(a_{3}-a_{4}\right) \text { and } 3=\left[b^{2}-f\left(a_{3}+a_{4}\right)^{2}\right]^{\frac{1}{2}} \text {. }
$$

Of course $p$ is real, because of the condition $4 b^{2}=\left(a_{3}: a_{4}\right)^{2}$. 
It might be thought that we could have cases to correspond to $\mathrm{I}(c), \mathrm{I}(d)$; but this is not so, on the ground of our restriction to reality.

Type III. Indices 211 .

$$
\begin{aligned}
& A=y_{1}^{2}+y_{2}^{2}+y_{3}^{2}-y_{4}^{2}, \\
& B=c_{1} y_{1}^{2}+c_{2} y_{2}^{2}+c_{3}\left(y_{3}^{2}-y_{4}^{2}\right) \pm\left(y_{3}-y_{4}\right)^{2},
\end{aligned}
$$

the roots of $|\lambda A-B|=0$ being $\lambda=c_{1}, c_{2}, c_{3}, c_{3}$.

$\operatorname{III}(a) . c_{1}, c_{2}, c_{3}$ all different; represented by [211]. This gives the elliptic and hyperbolic paraboloids* (C., p. 166).

$\operatorname{III}(b) . c_{2}=c_{3}$; represented by $[(21) 1]$. This is the horocyclic paraboloid. (C., p. 167).

III $(c) . c_{1}=c_{2}=c_{3}$; represented by $[(211)]$. This is the horocyclic surface of zero curvature (C., p. 170); in this case the arbitrary sign must be positive, to avoid an ideal surface.

$\operatorname{III}(d) . \quad c_{1}=c_{2}$; represented by $[2(11)] . \quad$ This gives the paraboloids of revolution (C., p. 169).

$$
\begin{gathered}
\text { Type IV. Indices } 31 . \\
A=y_{1}^{2}+y_{2}^{2}+y_{3}^{2}-y_{1}^{2}, \\
B=c_{1} y_{1}^{2}+c_{2}\left(y_{2}^{2}+y_{3}^{2}-y_{4}^{2}\right)+2 y_{2}\left(y_{3}-y_{4}\right), \quad|B|=-c_{1} c_{2}^{3},
\end{gathered}
$$

the roots of $|\lambda A-B|=0$, being $c_{1}, c_{2}, c_{2}, c_{2}$.

IV $(a) . c_{1} \gtrless c_{2}$; represented by [31]. This is a surface to which Coolidge has not assigned a name; it is the first on p. 167 (C.). The equation given there contains two superfluous constants $a_{2}, a_{3} ; a_{2}$ may be replaced by 1 , and $a_{3}$ by 0 , without loss of generality. $\dagger$

* Again I do not stop to give the sub-classificatious. But it may be well to point out that the coefficients $a_{3}, a_{4}$ in CooLIDGE's equation are not invariants; the essentials are $c_{3}=\frac{1}{2}\left(a_{3}-a_{4}\right)$ and the sign of $\left(a_{3}-a_{4}\right)$. For if $a_{3}-a_{4}=2 e q^{2}$, where $e= \pm 1$, and $q$ is real, we can write $y_{3}-y_{4}=q\left(x_{3}-x_{4}\right), y_{3}+y_{4}=\left(x_{3}+x_{4}\right) / q$ and then $y_{3}^{2}-y_{4}^{2}=x_{3}^{2}-x_{4}^{2}$, while

$$
a_{3} x_{3}^{2}+\left(a_{4}-a_{3}\right) x_{3} x_{4}-a_{4} x_{4}^{2}=c_{3}\left(y_{3}^{2}--y_{4}^{2}\right)+e\left(y_{3}-y_{4}\right)^{2} .
$$

Similarly in CoolidaE's type for III $(b)$, the constant $a$ is superfluous, except as to sign ; in the type for III $(c), a$ must be positive and may be put equal to +1 . Then

†To prove this statement, call Coolidge's equation $B ; A$ being the equation to the absolute.

$$
B-A=\left(a_{1}-1\right) x_{1}^{2}+\left(x_{3}-x_{4}\right)\left[2 a_{2} x_{2}+a_{3}\left(x_{3}-x_{4}\right)\right]=\left(a_{1}-1\right) x_{1}^{2}+2 y_{2}\left(y_{3}-y_{1}\right),
$$

provided that $2 a_{2} y_{2}=2 a_{2} x_{2}+a_{3}\left(x_{3}-x_{4}\right), y_{3}-y_{4}=a_{2}\left(x_{3}-x_{4}\right)$. Now substitute in $A$ for $x_{2}$ in terms of $y_{2}, y_{3}, y_{4}$; thus we find

$$
A=x_{1}^{2}+y_{2}^{2}+\left(y_{3}-y_{4}\right)\left[-a_{33}^{a_{2}^{2}}+\frac{a_{3}^{2}}{4 a_{2}^{4}}\left(y_{3}-y_{4}\right)+\frac{1}{a_{2}^{2}}\left(x_{3}+x_{4}\right)\right] .
$$

Call the last expression in square braokets $\left(y_{3}+y_{4}\right)$, then

$$
A=x_{1}^{2}+y_{2}^{2}+\iota_{3}^{2}-y_{4}^{2}, \quad B-A=\left(a_{1}-1\right) r_{1}^{2}+2 y_{2}\left(y_{: 3}-y_{4}\right) \text {. }
$$


IV $(b) . c_{1}=c_{2}$; represented by $[(31)]$. This is the third surface considered on p. 167 (C.); in the equation there given we may take $r=2$ without loss of generality.

We have now exhausted all possible quadric surfaces, in hyperbolic space. There are thus twelve fundamental classes, represented by the symbols given in the table

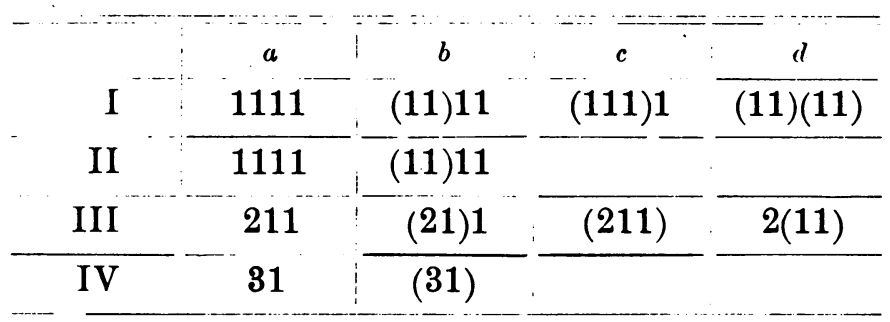

This classification can be completely carried out by an examination of $|\lambda A-B|$, without any preliminary reduction. But, for the further subdivisions, a certain amount of reduction is necessary ; as it is not possible to foretell in I which of the four roots $c_{1}, c_{2}, c_{3}, c_{4}$ is to be associated with the negative term in $A$. More can be done in III ; for $c_{3}$ is necessarily associated with the negative square; but here the sign of $\left(y_{3}-y_{4}\right)^{2}$ cannot be foretold. In cases II and IV we can obtain complete information from the determinant; as may be seen by considering the reduced forms.

\section{\$3. Quadrics in elliptic space.}

The absolute is here a positive quadratic form and its signature is 4 ; hence the only indices possible are 1111 , and the roots $c_{1}, c_{2}, c_{3}, c_{4}$ are all real.* Thus we are restricted to the possibilities given by the row numbered $I$ in the table at the end of $\$ 2$.

IIence every quadric in elliptic space can be reducerl to the form

$$
c_{1} y_{1}^{2}+c_{2} y_{2}^{2}+c_{3} y_{3}^{2}+c_{4} y_{4}^{2}=0
$$

the absolute being $y_{1}^{2}+y_{2}^{2}+y_{3}^{2}+y_{1}^{2}=0$; the coefficients $c_{1}, c_{2}, c_{3}, c_{4}$ are all real, being the roots of $|\lambda A-B|=0$.

The details of the cases are :

$I(a)$. [1111] ; surficces not of revolution, ruled or not according as the product $c_{1} c_{2} c_{3} c_{4}$ is positive or negative.

$\mathrm{I}(b) \cdot[(11) 11]$; surfuces of recolution $\left(c_{1}=c_{2}\right)$, ruled or not according as $c_{3} c_{4}$ is positive or negative.

$\mathrm{I}(c) .[(111) 1] ;$ spheres (not ruled), $c_{1}=c_{2}=c_{3}$.

* This fact follows also from two theorems due to Weierstrass (Berliner Monatsberichte, f858, p. 207 ; (iestemmelte $W^{\prime}{ }^{\prime}(k)$, vol. 1, p. 233 ; the theorems are given in $\$ \$ 3,4$ ). 
$\mathrm{I}(d) \cdot\left[(11 ;(11)]\right.$; Clifford's surfaces, (ruled) $c_{1}=c_{2}, c_{3}=c_{4}$.

It may be remarked that there are no hyperboloids in any of these cases.

\section{\$4. Quadrics in parabolic (Euclidean) space.}

The classification of quadrics in Euclidean space is carried out in most of the ordinary text-books on solid geometry, and details need hardly be given here: but a few remarks may be added, to indicate the contrast to the cases considered in $\S \S 2,3$.

In parabolic space the absolute is degenerate; its equation can be expressed only in terms of plane-coördinates $u_{1}, u_{2}, u_{3}, u_{4}$. If then $A=0$, is the alssolute, it will be found that the determinant $|A|=0$, and that the signature of $A$ is 3 . Thus, if $B=0$ is any quadric (in plane-coördinates) the determinant* $|\lambda A+\mu B|$ has

either (i) four linear invariant-factors $\lambda+\mu c_{1}, \lambda+\mu c_{2}, \lambda+\mu c_{3}, \mu$ :

or (ii) two linear invariant-factors $\lambda+\mu c_{1}, \lambda+\mu c_{2}$ and one $\mu^{2}$ :

where, in each case, $c_{1}, c_{2}, c_{3}$ (or $c_{1}, c_{2}$ ) are real.

From these facts we see that, by applying Weiers'rRass's methods (in conjunction with a slight modification $\dagger$ so as to include the factors $\mu$, or $\mu^{2}$ ), the reduced types are

$$
\begin{aligned}
& \left\{\begin{array}{l}
A=v_{1}^{2}+v_{2}^{2}+v_{3}^{2}, \\
B=c_{1} v_{1}^{2}+c_{2} v_{2}^{2}+c_{3} v_{3}^{2}+c_{4} v_{4}^{2},
\end{array}\right. \\
& \left\{\begin{array}{l}
A=v_{1}^{2}+v_{2}^{2}+v_{3}^{2}, \\
B=c_{1} v_{1}^{2}+c_{2} v_{2}^{2}+2 f_{v_{3}} v_{4} .
\end{array}\right.
\end{aligned}
$$

Here the coefficients $c_{4}, f$ cannot be found from the determinant $|\lambda A+\mu B|$; it is not usually necessary to find them merely for the purpose of classification. $f$ But they must be found if we require the precise size of the surface $B$ : one method has been given by the present writer elsewhere. $\S$

Another possibility may occur if $|Z|=0$; it may happen that

$$
|\lambda A+\mu B|=0,
$$

\footnotetext{
* Either by the modified form of KLEIx's theorem given above ; or by a theorem due to GUN DELFINGER which is given in his edition of Hesse's lectures on geometry and also in his lectures on conics (p. 67). See MUTI's Elemirutartheiler, p. 180.

† Proceedings of the London Mathematical Society, vol. 32 (1900), p. 158.

$\mp$ To distinguish hetween ruled and nop-ruled surfaces, the sign of $|B|$ is suflicient

\$ Proceedings of the Cambridge Philosophical Society, vol. 10)(1901), p. 358 (see ? 3) ; see also BR ̈̈̈CKEL's collection of GundeLFingkr's formulae in Crelle's Journal für die II athematik, vol. 119 (1898), pp. $210,313$.
} 
identically. If so, the reduced forms are

$$
\left\{\begin{array}{l}
A=v_{1}^{2}+v_{2}^{2}+v_{3}^{2}, \\
B=c_{1} v_{1}^{2}+c_{2} v_{2}^{2}+c_{3} v_{3}^{2},
\end{array}\right.
$$

$B$ being a conic in the plane at infinity.

But if a quadric given in point-coördinates is degenerate the method just explained breaks down entirely; for the transformation of such an equation to plane-cördinates is no longer possible. * To meet this difficulty an alternative method of reduction is explained in $\$ \S 1,2$ of the paper just quoted; and a simple general method of classification is obtained. But the process by which the result is obtained is not very easy; and it may therefore be worth while to state the result in order to make it accessible to those who do not care to go through the details which are unavoidable in finding the reducing substitution. Let $R=0$ be the given quadric, expressed in point-coördinates $x_{1}, x_{2}, x_{3}, x_{4}$; $\dot{S}=0$ any sphere, the coefficients of $S$ having been so chosen that the expression $S /\left(p_{1} x_{1}+p_{2} x_{2}+p_{3} x_{3}+p_{4} x_{4}\right)^{2}$ represents the square of the tangent from $\left(x_{1}, x_{2}, x_{3}, x_{4}\right)$ to the sphere, where $p_{1} x_{1}+p_{2} x_{2}+p_{3} x_{3}+p_{4} x_{4}=0$ is the plane at infinity. Write $\Delta_{0}$ for the determinant $|\lambda R-S|$ : and $\Delta_{1}$ for the symmetrical determinant formed by adding a fifth row and column $\left(p_{1}, p_{2}, p_{3}, p_{1}, 0\right)$ to $\Delta_{0}$. Then the elements of classification are $\dagger$ :

(i) the factors of $\Delta_{1}$; say $1-\lambda c_{1}, 1-\lambda c_{2}, 1-\lambda c_{3}$; where $c_{1}, c_{2}, c_{3}$ are all real and some may be zero.

(ii) the first term (say $\left.k \lambda^{a}\right)$ in the expansion of $\left(-\Delta_{0} / \Delta_{1}\right)$ in descending powers of $\lambda$.

When the quadric is transformed to orthogonal Cartesian coördinates (say $\left.y_{1}, y_{2}, y_{3}\right)$, the terms of the second degree can be reduced to

$$
c_{1} y_{1}^{2}+c_{2} y_{2}^{2}+c_{3} y_{3}^{2}
$$

and, as to the remaining terms, three cases are possible, according to the value of the integer $\alpha$. To put the work in as short a form as possible, we assume (from the results of the elementary investigations) that $R, S$ must take the forms

$$
\begin{aligned}
& R=c_{1} y_{1}^{2}+c_{2} y_{2}^{2}+c_{3} y_{3}^{2}+2 f y_{3}+c_{4}, \\
& S=y_{1}^{2}+y_{2}^{2}+y_{3}^{2}+2\left(l_{1} y_{1}+l_{2} y_{2}+l_{3} y_{3}\right)+m,
\end{aligned}
$$

\footnotetext{
* More precisely, the transformed equation either vanishes identically or fails to give sufficient information. It should be observed that the corresponding difficulty does not arise in hyperbolic or elliptic space ; for the absolute may be expressed either in point or in plane-coürdinates.

$\dagger$ It is the second of these which constitutes the chief simplification introduced by this method of classification.
} 
where, in $R$, either $f$ is zero, or both $c_{3}, c_{4}$ are zero. It is then easy to see that

$$
\begin{aligned}
\Delta_{1} & =-M^{2}\left(\lambda c_{1}-1\right)\left(\lambda c_{2}-1\right)\left(\lambda c_{3}-1\right), \\
\Delta_{0}=M^{2}\left[\left(\lambda c_{1}-1\right)\right. & \left(\lambda c_{2}-1\right)\left(\lambda c_{3}-1\right)\left(\lambda c_{4}-m\right)-l_{1}^{2}\left(\lambda c_{2}-1\right)\left(\lambda c_{3}-1\right) \\
& \left.-l_{2}^{2}\left(\lambda c_{3}-1\right)\left(\lambda c_{1}-1\right)-\left(f \lambda-l_{3}\right)^{2}\left(\lambda c_{1}-1\right)\left(\lambda c_{2}-1\right)\right],
\end{aligned}
$$

$M$ being the determinant of a certain linear substitution. From this we find that $c_{1}, c_{2}, c_{3}$ are the letters obtained in (i) from the factors of $\Delta_{1}$; also that

$$
-\frac{\Delta_{0}}{\Delta_{1}}=\lambda c_{4}-m-l_{1}^{2}-\lambda c_{1}-1-\lambda c_{2}^{2}-1-\frac{\left(f \lambda-l_{3}\right)^{2}}{\lambda c_{3}-1} .
$$

Now, according to (ii), $k \lambda^{a}$ is the first term of this expression when expanded in powers of $1 / \lambda$; thus, we have three cases:

$$
\begin{aligned}
& f=0 ; \text { then } k \lambda^{a}=\lambda c_{4} ; \text { so that } k=c_{4}, \alpha=1, \\
& f^{*}=0, c_{4}=0 ; \text { here } \alpha<1, \\
& c_{3}=0, c_{4}=0 \text {; then } k \lambda^{a}=\left(f^{2} \lambda\right)^{2} ; \text { so that } k=f^{2}, \alpha=2 .
\end{aligned}
$$

Thus, classifying by means of the index $\alpha$, we may write the results

$$
\begin{array}{ll}
\alpha=1, & R=c_{1} y_{1}^{2}+c_{2} y_{2}^{2}+c_{3} y_{3}^{2}, \\
\alpha=1, & R=c_{1} y_{1}^{2}+c_{2} y_{2}^{2}+c_{3} y_{3}^{2}+k, \\
\alpha=2, & R=c_{1} y_{1}^{2}+c_{2} y_{2}^{2}+2 k_{2}^{2} y_{3},
\end{array}
$$

giving a complete classification of all quadrics * in point-coördinates.

Queen's Collece, Galway, February 22, 1904.

Addition. Mr. Coolidge has kindly recalled to my memory the fact that a classification of quadrics, in hyperbolic and elliptic space, had been previously given by P. BARBarin. $\dagger$ In this classification of hyperbolic space, the classes I, II, III, are the same as the classes which bear these numbers in $\$ 2$ above; but class IV is left out. The omission is due to an oversight in connection with the transformations given at the top of p. 113 (1. c.), $\$ 28$; in fact if the absolute is $x^{2}+y^{2}+z^{2}-u^{2}=0$, the quadric $z^{2}+2 x(y-u)=0$ has only one real principal plane $(z=0)$; and this quadric cannot be reduced to BARBARIN's typical equation (40), $S_{1} X^{2}+S_{2} y^{2}+S_{2} Z^{2}-2 P U X=0$. There is a similar difficulty with any quadric belonging to class IV of $\$ 2$.

* It may be worth while to point out that if the quadric is degenerate one or more of $c_{1}, c_{2}, c_{3}$ may be zero; but this involves no change in the calculation of $c_{4}$ and $f$.

†'Études de Géometrie Non-Fuclidienne, Bruxelles, 1900, pp. 101-126; this is an extract (published separately) from vol. 60 of the $M$ émoires couronnés et autres Mémoires publiés par l'Académie royale de Belgique. 
It is to be noticed that BARBARIN's nomenclature differs in some respects from Coolidge's, which has been used above; the former gives the generic name paraboloid to class II, and horiquadric to class III. Although there is an advantage in giving a name to each class, yet it does not appear to me that the name paraboloid is specially suited to class II, the quadrics of which have imaginary centers.

In conclusion, the classification of conics in the hyperbolic plane, carried out on the lines of $\S \S 1,2$, is :

$$
\begin{array}{ll}
\mathrm{I}(a): & {[111] ; c_{1} y_{1}^{2}+c_{2} y_{2}^{2}-c_{3} y_{3}^{2}=0 .} \\
(b): & {[(11) 1] ; c_{1}\left(y_{1}^{2}+y_{2}^{2}\right)-c_{3} y_{3}^{2}=0, \text { and } c_{1} y_{1}^{2}+c_{2}\left(y_{2}^{2}-y_{3}^{2}\right)=0 .} \\
(c): & {[(111)] ; y_{1}^{2}+y_{2}^{2}-y_{3}^{2}=0 \text { (the absolute). }}
\end{array}
$$

II: $\quad[\overline{11} 1] ; c_{1} y_{1}^{2}+\alpha\left(y_{2}^{2}-y_{3}^{2}\right)+2 \beta y_{2} y_{3}=0$.

III $(a): \quad[21] ; c_{1} y_{1}^{2}+c_{2}\left(y_{2}^{2}-y_{3}^{2}\right) \pm\left(y_{2}-y_{3}\right)^{2}=0$.

(b): $\quad[(21)] ; c_{1}\left(y_{1}^{2}+y_{2}^{2}-y_{3}^{2}\right) \pm\left(y_{2}-y_{3}\right)^{2}=0$.

IV :

$$
\text { [3]; } c_{1}\left(y_{1}^{2}+y_{2}^{2}-y_{3}^{2}\right)+2 y_{1}\left(y_{2}-y_{3}\right)=0 \text {. }
$$

In each case the absolute is $y_{1}^{2}+y_{2}^{2}-y_{3}^{2}=0$; and, according to KLeIN's theorem, there is at least one real invariant factor of odd degree.

The numbering of classes I, II, III corresponds to that of BARBARIN (l. c. pp. 39-57), who omits class IV; in fact the method given on p. 33 for determining the rotation $\theta$ fails if applied to the conics IV; and these conics cannot be reduced to the form contained in BARBARn's equation $\left(20_{1}\right)$ on p. 40, which is the basis of his subsequent work.

It is of course possible to give a classification of conics in the elliptic and in the parabolic planes, by a suitable modification of the work above; but the necessary changes are so obvious that it is hardly worth while to enumerate them.

March 15, 1805 